\title{
Farmácia: a porta de entrada para o acesso a medicamentos para idosos residentes em Santos
}

\section{Pharmacy: a gateway to access to medicines for elderly individuals living in the city of Santos}

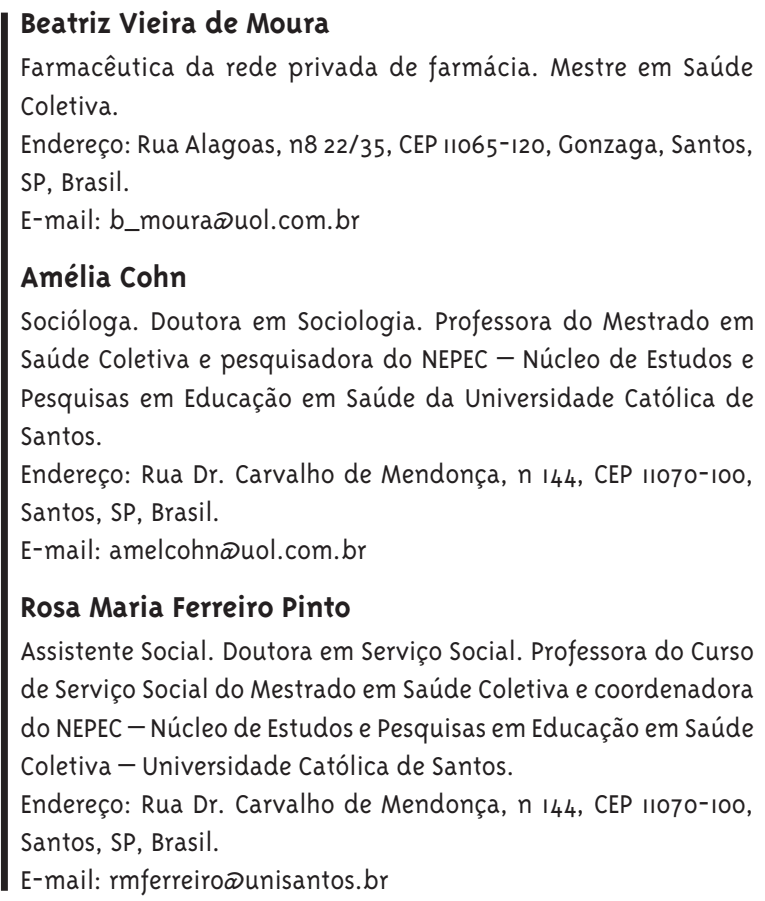

Assistente Social. Doutora em Serviço Social. Professora do Curso de Serviço Social do Mestrado em Saúde Coletiva e coordenadora do NEPEC - Núcleo de Estudos e Pesquisas em Educação em Saúde Coletiva - Universidade Católica de Santos.

Endereço: Rua Dr. Carvalho de Mendonça, n 144, CEP 11070-100, Santos, SP, Brasil.

E-mail: rmferreiro®unisantos.br

\section{Resumo}

Este estudo teve como objetivos conhecer o perfil dos medicamentos utilizados pelos idosos, com ênfase na associação entre aqueles de uso contínuo mais utilizados e outros fármacos sem indicação médica e avaliar a função social do farmacêutico nas farmácias privadas. 0 método utilizado foi a pesquisa qualitativa de natureza compreensiva e explicativa, realizada no município de Santos, em 2008, em bairros com maior concentração de idosos (IBGE,200o). Nesses bairros, foram identificadas três farmácias privadas, que autorizaram a realização da pesquisa por um período de uma semana, quando foram realizadas as entrevistas, a partir de um roteiro estruturado.Os resultados evidenciaram um maior consumo dos idosos para as classes farmacológicas dos medicamentos de uso contínuo (os anti-hipertensivos, antidiabéticos, diuréticos e o antiagregante plaquetário). Dentre os medicamentos adquiridos sem prescrição médica, destacaram-se a compra de: analgésicos/antitérmicos, antigripais, antiinflamatórios e fitoterápicos. Alguns dos que compõem esse conjunto são tarjados e exigiriam a apresentação do receituário. As evidências obtidas apontam para o fato de que o consumo autoindicado, associado ao uso de medicamentos de uso contínuo, é uma prática rotineira entre os idosos.

Palavras-chave:Idosos; Uso de medicamentos; Medicamento sem prescrição médica. 


\section{Abstract}

This study aimed to profile the consumption of drugs by seniors, with emphasis on the association between the most commonly purchased drugs of continueduse and other drugs without medical prescription, and to evaluate the social role of the pharmacist in private pharmacies.The method was qualitative research, of the comprehensive and explanatory type, carried outin the city of Santos in 2008 , inneighborhoods with high concentration of elderly people(IBGE 2000). In these neighborhoods, three private pharmacieswere identified.They authorized the conduction of the research for one week. During this period,interviews were performedbasedon a structured script.The results showed that the elderly mostly consumethe pharmacological classes of drugs ofcontinuous use: antihypertensives, antidiabetic drugs, diuretics and the antiplatelet agent.The main drugs acquired without medical prescription were: analgesics and antipyretics, anti-flu drugs, antiinflammatory drugs and herbal medicines. Some of those who comprise this set could not have been acquired without prescription. The evidences point to the fact that self-prescribedconsumption, associated with the utilizationof drugs of continuous use, is a common practice among the elderly.

Keywords: Elderly; Use of Drugs; Medicine Without Prescription.

\section{Introdução}

Frente às mudanças do padrão demográfico brasileiro, dentre as quais serem os idosos o segmento etário que proporcionalmente mais cresce no Brasil, o uso de medicamentos por eles passa a ganhar relevância no campo da Saúde Coletiva. Isso porque seu padrão de consumo está associado principalmente às doenças de maior prevalência nessa faixa etária, como as cardiovasculares e diabetes, tornando-se necessário levar-se em conta a possibilidade do desenvolvimento de interações farmacológicas indesejáveis, posto que a polifarmácia é uma das consequências da multiplicidade de patologias que no geral apresentam, associada à procura de outros medicamentos não prescritos, dado que os estabelecimentos que os comercializam disponibilizam essa mercadoria enquanto tal: uma mercadoria a ser consumida.

Observa-se, assim, que de um lado essa faixa etária está exposta simultaneamente a fatores como o excesso de prescrições por parte dos médicos, e por outro ao consumo de medicamentos não prescritos, utilizados concomitantemente com os de uso contínuo. Ambas as classes de fatores contribuem para um padrão de consumo de medicamentos aparentemente "irracional" pelos idosos, mas que tem sua lógica e que de qualquer forma gera consequências que acabam onerando os serviços de saúde, dada a ocorrência potencial ou efetiva de efeitos colaterais indesejáveis.

No entanto, como o acesso a medicamentos tem por finalidade específica a resolutividade das ações de saúde e o bem-estar do paciente por meio de garantia de dosagem correta e por tempo adequado, isso exige que esse consumo esteja baseado na sua utilização "racional", isto é, segundo as prescrições médicas e farmacológicas. Nesse ponto é que ganha destaque o papel do (a) farmacêutico (a), ou dos profissionais de farmácia, para que garantam o perfil "saudável" ou "racional" desse tipo de consumo pelos idosos, fazendo com que seja superada a concepção de consumo de medicamento como uma mera relação de compra e distribuição; que seja racionalizado o uso de medicamentos; e que sejam reduzidos os riscos associados à automedicação. Em resumo: cumpre a eles assumir a responsabilidade com a farmacoterapia e articular as farmácias aos serviços do SUS. Assim, a participação do farmacêu- 
tico na dispensação e orientação de medicamentos à população, e principalmente aos idosos, torna-se fundamental para contribuir com as diretrizes e prioridades da Política Nacional de Medicamentos (Brasil, 2001) quanto à Promoção do Uso Racional de Medicamentos. Nesse contexto, a atenção farmacêutica constitui-se numa das ações fundamentais através do compromisso desse profissional com a sua responsabilidade pela definição das necessidades farmacoterápicas do paciente, pois é através da orientação farmacêutica que o farmacêutico torna-se corresponsável pela qualidade de vida do paciente.

Dentre essas responsabilidades e compromissos, estão as ações voltadas para se evitar a prática da automedicação, uma vez que dela decorrem vários problemas de saúde, já bastante conhecidos. E se a automedicação é uma prática mundial, no Brasil as políticas públicas específicas vêm-se revelando frágeis e pouco atuantes para inibir essa prática, exigindo que um conjunto de ações e estratégias seja acionado de forma articulada para que a conduta de autoconsumo de medicamentos no Brasil seja reduzida. Exatamente nesse ponto ações junto aos estabelecimentos de venda de medicamentos ganham importância, uma vez que a farmácia acaba se tornando, na maioria dos casos, a porta de entrada para o acesso aos medicamentos, tanto prescritos como os de autoconsumo. Daí vários autores defenderem que os estabelecimentos que comercializam medicamentos se constituam, dada sua natureza, num centro prestador de serviço público (Santos, 1998a), e ainda que se integrem as farmácias ao Sistema Sanitário brasileiro, com o intuito de que contribuam na atenção à saúde, através da participação em programas de prevenção e promoção de saúde (Zubioli,1996; Santos, 1998).É, portanto,nas farmácias que o trabalho do farmacêutico se revela essencial na atenção à saúde, oferecendo seus conhecimentos farmacológico-clínicos cientificamente embasados para orientação dos pacientes, sejam eles de origem pública ou privada. É aí que o farmacêutico concretiza seu compromisso com a saúde da população, garantindo a orientação com qualidade no momento do acesso e da dispensação do medicamento.

A inexistência de estudos a respeito do uso inde- vido de medicamentos por pessoas idosas na cidade de Santos levou ao desenvolvimento deste estudo voltado ao conhecimento do perfil de consumo dos medicamentos mais utilizados por idosos, os medicamentos de uso contínuo e prescritos e aqueles não prescritos, podendo levar à ocorrência de possíveis efeitos indesejáveis.

\section{Método e Técnica de Pesquisa}

A pesquisa foi realizada junto a 95 sujeitos idosos, no ano de 2008, em farmácias localizadas em três bairros de Santos (Embaré, Boqueirão e Aparecida), os quais apresentam maior concentração de residentes com 60 anos ou mais de idade (IBGE, 200o). Também foram entrevistados os profissionais farmacêuticos dos estabelecimentos onde se desenvolveu a pesquisa. Cabe salientar que esses são bairros localizados na orla da praia, região do município que apresenta melhores indicadores sociais (SEADE, 2000; IBGE, 2000).

Foi realizado um estudo qualitativo de natureza exploratória, visando à tentativa de descrever, compreender e explicar aspectos do recorte da realidade de uma parte da população a ser investigada em determinado espaço e tempo histórico, com os seguintes objetivos: conhecer o perfil de consumo dos medicamentos utilizados por idosos e avaliar a importância do farmacêutico na dispensação e orientação.

A pesquisa contou com as seguintes etapas: escolha dos estabelecimentos nos bairros predeterminados, mediante a solicitação e autorização; seleção de uma farmácia em cada bairro; pesquisa de campo (realização de entrevistas com consumidores e profissionais farmacêuticos). As farmácias em que a pesquisa foi realizada são as denominadas "farmácias de bairro", já que os estabelecimentos das grandes redes foram contatados, mas nenhum deles autorizou a realização da coleta de dados. Se, por um lado, esse foi um aspecto a princípio considerado como negativo, por outro, os estabelecimentos pesquisados revelaram maior proximidade entre os consumidores e os funcionários.

Os sujeitos da pesquisa foram 95 idosos - homens e mulheres -com idade igual ou superior a 60 anos. A escolha dos idosos foi acidental e aleatória, de 
acordo com sua presença nos períodos e horários determinados pelos estabelecimentos para a realização da pesquisa. A média de permanência da pesquisadora em cada estabelecimento foi de uma semana, quando então também foram realizadas as entrevistas com os farmacêuticos dos respectivos estabelecimentos.

As informações foram obtidas através da abordagem direta e do convite para participar da pesquisa, seguindo-se os procedimentos de natureza ética exigidos por pesquisas dessa natureza. A coleta de dados foi realizada através de entrevista no local, utilizando-se de questionários pré-testados (um para o usuário e outro para o profissional), composto de questões estruturadas.

Algumas das principais perguntas abordadas no questionário para os idosos apresentam-se na forma de blocos.

$\mathrm{O}_{1}{ }^{\circ}$ bloco refere-se aos dados pessoais de identificação:

1. Dados pessoais de identificação:

\begin{tabular}{|l|}
\hline Nome completo: \\
\hline Endereço: \\
\hline Bairro: \\
\hline Data de nascimento: \\
\hline Sexo:
\end{tabular}

$\mathrm{O} 2^{\circ}$ bloco refere-se aos indicadores sociais:

\begin{tabular}{|lcc|}
\hline I. Faixa etária: & & \\
\hline Idade & Homens & Mulheres \\
\hline 60 a 67 & $26 \%$ & $28 \%$ \\
\hline 68 a 75 & $36 \%$ & $42 \%$ \\
\hline 76 a + & $38 \%$ & $30 \%$ \\
\hline
\end{tabular}

\begin{tabular}{|lcccc|}
\hline \multicolumn{4}{|l|}{ 2. Distribuição do número dos sujeitos por sexo e bairro: } & \\
\hline Bairros & Boqueirão & Aparecida & Embaré & Total \\
\hline Homens & 17 & 11 & 14 & 42 \\
\hline Mulheres & 21 & 13 & 19 & 53 \\
\hline Total de sujeitos & 38 & 24 & 33 & 95 \\
\hline
\end{tabular}

\begin{tabular}{|lll|}
\hline 3. Grau de escolaridade: & Homens & Mulheres \\
\hline 18 grau incompleto & $19 \%$ & $17 \%$ \\
\hline 18 grau completo & $24 \%$ & $62 \%$ \\
\hline 28 grau incompleto & $2 \%$ & $6 \%$ \\
\hline 28 grau completo & $36 \%$ & $9 \%$ \\
\hline Nível técnico & $5 \%$ & $4 \%$ \\
\hline Nível superior completo & $14 \%$ & $2 \%$ \\
\hline
\end{tabular}

\begin{tabular}{|lcccc|}
\hline 4. Estado civil: & Casado & Solteiro & Divorciado & Viúvo \\
\hline Homens & $74 \%$ & $2 \%$ & $12 \%$ & $12 \%$ \\
\hline Mulheres & $36 \%$ & $13 \%$ & $8 \%$ & $43 \%$ \\
\hline
\end{tabular}




\begin{tabular}{|lcccc|}
\hline 5. Renda mensal: & I S.M. & 2 a 4 S.M. & 5 a 7 S.M. & 7 a + S.M. \\
\hline Homens & $2 \%$ & $64 \%$ & $17 \%$ & $17 \%$ \\
\hline Mulheres & $15 \%$ & $76 \%$ & $9 \%$ & $0 \%$ \\
\hline
\end{tabular}

\begin{tabular}{|lcc|}
\hline 6. Exerce atualmente atividade profissional: & \\
\hline Bairros & Homens & Mulheres \\
\hline Boqueirão & $42 \%$ & $20 \%$ \\
\hline Aparecida & $25 \%$ & $40 \%$ \\
\hline Embaré & $33 \%$ & $40 \%$ \\
\hline
\end{tabular}

$\mathrm{O} 3^{\circ}$ bloco refere-se ao questionário com as principais perguntas abordadas junto aos idosos nos três bairros:

\begin{tabular}{|lcc|}
\hline I. Frequência aproximada à visita médica: & Homens & Mulheres \\
\hline Semanal & $0 \%$ & $0 \%$ \\
\hline Mensal & $2 \%$ & $2 \%$ \\
\hline Bimestral & $2 \%$ & $6 \%$ \\
\hline Trimestral & $17 \%$ & $55 \%$ \\
\hline Semestral & $41 \%$ & $24 \%$ \\
\hline Anual & $38 \%$ & $13 \%$ \\
\hline
\end{tabular}

\begin{tabular}{|lcc|}
\hline 2. Plano de saúde privado: & Homens & Mulheres \\
\hline Sim & $69 \%$ & $81 \%$ \\
\hline Não & $31 \%$ & $19 \%$ \\
\hline
\end{tabular}

\begin{tabular}{|lcc|}
\hline 3. Uso de medicação de uso contínuo: & & \\
\hline Bairros & Homens & Mulheres \\
\hline Boqueirão & $50 \%$ & $42 \%$ \\
\hline Aparecida & $16 \%$ & $22 \%$ \\
\hline Embaré & $34 \%$ & $36 \%$ \\
\hline
\end{tabular}

\begin{tabular}{|lcc|}
\hline 4. Procurar a farmácia antes de ir ao médico: & \\
\hline Bairros & Homens & Mulheres \\
\hline Boqueirão & $42 \%$ & $20 \%$ \\
\hline Aparecida & $35 \%$ & $44 \%$ \\
\hline Embaré & $23 \%$ & $36 \%$ \\
\hline
\end{tabular}

5. Não solicitaram orientação farmacêutica:

\begin{tabular}{|lcc|}
\hline Bairros & Homens & Mulheres \\
\hline Boqueirão & $33 \%$ & $49 \%$ \\
\hline Aparecida & $27 \%$ & $21 \%$ \\
\hline Embaré & $40 \%$ & $30 \%$ \\
\hline
\end{tabular}


6. Consumo de medicamentos não prescritos:

Homens $55 \%$

Mulheres $38 \%$

7. Esquecimento dos medicamentos de uso contínuo:

Homens $57 \%$

Mulheres $45 \%$

8.Confusão na tomada dos medicamentos de uso contínuo:

Homens $28 \%$

Mulheres $24 \%$

\begin{tabular}{|lcc|}
\hline 9. Gastos com medicamentos de uso contínuo: & Homens & Mulheres \\
\hline Abaixo da renda mensal & $72 \%$ & $62 \%$ \\
\hline Acima da renda mensal & $0 \%$ & $0 \%$ \\
\hline $10 \%$ da renda mensal & $19 \%$ & $17 \%$ \\
\hline $20 \%$ da renda mensal & $5 \%$ & $8 \%$ \\
\hline $30 \%$ da renda mensal & $2 \%$ & $13 \%$ \\
\hline $40 \%$ da renda mensal & $2 \%$ & $0 \%$ \\
\hline $50 \%$ da renda mensal & $0 \%$ & $0 \%$ \\
\hline
\end{tabular}

$\mathrm{O} 4^{\circ}$ bloco refere-se aos dados da empresa:

I. Dados da Empresa:

Razão social

CNPJ

Inscrição estadual

Nome fantasia

O bloco $5^{\circ}$ refere-se aos dados de identificação dos farmacêuticos:

Nome completo:

CRF

O bloco $6^{\circ}$ refere-se a algumas perguntas efetuadas aos farmacêuticos:

\begin{tabular}{|ll|}
\hline I. Informações sobre o uso racional de medicamentos aos clientes: \\
\hline Bairros & $50 \%$ \\
\hline Boqueirão & $17 \%$ \\
\hline Aparecida & $33 \%$ \\
\hline Embaré &
\end{tabular}


2. Medicamentos indicados por:

\begin{tabular}{|ll|}
\hline Médico & $40 \%$ \\
\hline Farmacêutico & $12 \%$ \\
\hline Amigo/Conhecido & $12 \%$ \\
\hline Propaganda & $12 \%$ \\
\hline Autoindicado & $24 \%$ \\
\hline
\end{tabular}

\section{Resultados e Discussão}

A cidade de Santos está localizada no litoral do Estado de São Paulo, a $72 \mathrm{~km}$ da capital, tem seus limites junto às cidades de Santo André, Mogi das Cruzes e Salesópolis ao Norte, com o oceano Atlântico e a Ilha de Santo Amaro ao Sul (Guarujá), com Bertioga ao Leste e Cubatão e São Vicente à Oeste. Latitude $23^{\circ} 56^{\prime} 26^{\prime}$ "sul e longitude $46^{\circ} 19^{\prime} 47^{\prime \prime}$ oeste. A população está estimada em 417.983 hab. (IBGE, 200o). Possui uma área total de $271 \mathrm{~km}^{2}$, área insular de39 km² e área continental de $232 \mathrm{~km}^{2}$.

O município de Santos possui aproximadamente 47 bairros. Os bairros de maior concentração de idosos, com idade igual ou acima de 60 anos de idade em Santos, são: Embaré (7.022), Boqueirão (6.995), Aparecida (6.944), Gonzaga (5.834), Ponta da Praia (5.144) e Marapé (IBGE, 200o). A pesquisa foi realizada em uma área privilegiada da cidade, em um setor de fácil acesso a todos os tipos de serviços e lazer.

O grupo pesquisado, em relação aos outros bairros deste município, apresentou um perfil socioeconômico relativamente alto, com alta escolaridade, e um perfil de acesso aos serviços de saúde assegurado fundamentalmente por meio da assistência privada à saúde. 0 segmento masculino no bairro Boqueirão foi maior que nos demais, cerca de $40 \%$. No entanto, entre os homens, muitos ainda exercem atividade profissional, possuem escolaridade até o $2^{\circ}$ grau completo e alguns com nível superior, renda de até sete salários mínimos, idade acima de 68 anos. No geral, apresentam um padrão de consumo maior de medicamentos prescritos e não prescritos e alto grau de esquecimento e confusão na tomada das medicações. Fato surpreendente é que apresentam maior frequência quanto a procurar a farmácia antes de se consultar com um médico ou um serviço médico.
Já no que diz respeito às mulheres, sua presença enquanto parcela da população nessa faixa etária foi mais equilibrada do que em relação ao grupo dos homens, nos três bairros, tendo maior representatividade do que os homens, confirmando estudos anteriores (Saldanha, 2004). No caso delas, a visita médica costuma ser realizada sistematicamente. Já em relação aos anos de estudo, a maioria das mulheres tinha $01^{\circ}$ grau completo, e, como era esperado, possuíam uma renda mensal menor, pois a maioria das entrevistadas já era viúva.

Em ambos os segmentos o consumo de medicamentos de uso contínuo apresentou-se como predominante, coincidindo com os problemas de saúde mais frequentes entre os idosos: hipertensão e diabetes. 0 gasto com esses medicamentos de uso contínuo apresentou-se abaixo da renda mensal, pois a maioria dos idosos faz opção por genéricos ou participa do programa "Farmácia Popular do Brasil" (Brasil, 2004). Considerando que a renda média nesse grupo é de até quatro salários mínimos, os gastos com medicamentos representaram aproximadamente $20 \%$ da renda mensal; no Brasil, segundo estudo baseado na Pesquisa Nacional por Amostra de Domicílios em 1998 (PNAD), 50\% dos idosos têm renda mensal menor que um salário mínimo e o gasto médio mensal com medicamentos compromete um quarto dessa renda.

Quanto às classes farmacológicas de medicamentos de uso contínuo mais utilizadas pelos idosos do sexo masculino, prevalecem os anti-hipertensivos e diuréticos. No caso das mulheres, os anti-hipertensivos e aqueles destinados ao tratamento de perda óssea (osteoporose), confirmando que as doenças mais prevalentes nessa faixa etária são as cardiovasculares e diabetes.

Dentre as classes farmacológicas mais consumidas pelos idosos sem prescrição médica estão os 
analgésicos e anti-inflamatórios, incluindo medicamentos de venda livre, medicamentos fitoterápicos, inclusive medicamentos tarjados, que exigem a apresentação do receituário médico ou odontológico, reafirmando com isso resultados de outras pesquisas, como o estudo de base populacional sobre o consumo de medicamentos entre idosos, em Bambuí (Loyola, 2005).

O questionário aplicado contemplava questões sobre esquecimento e confusão experimentados nas últimas semanas em relação aos horários e às frequências de se tomar os medicamentos prescritos. De fato, foi observado nas respostas um alto grau de esquecimento e confusões dos consumidores, devido à grande quantidade de medicamentos utilizados, ou seja, de três ou mais classes farmacológicas. Esse "déficit de memória" como fator de esquecimento e confusão apresentou-se maior entre os homens. Mesmo nos casos dos medicamentos de uso contínuo e diário, portanto nos casos que fazem parte de uma rotina terapêutica diária, observou-se entre os sujeitos um alto grau de esquecimento e confusão. Os bairros do Boqueirão e do Embaré apresentaram maior frequência do fator esquecimento, comparados com o bairro de Aparecida, com expressiva diferenciação entre idosos e idosas: o número total de sujeitos idosos (homens) dos três bairros somam 42 sujeitos, relacionando em valores percentuais, os idosos no bairro do Boqueirão apresentaram um percentual de $48 \%$, no bairro de Embaré o valor apresentado foi de $47 \%$; o bairro de Aparecida apresentou o menor percentual, $5 \%$.

Com as idosas o valor apresentado foi homogêneo se comparado com o dos idosos, sendo assim, no bairro Boqueirão apresentou o percentual de $27 \%$; no bairro de Aparecida 30\% e no Embaré 43\%. Já em relação ao fator confusão, os índices encontrados foram altos, o percentual apresentado para homens no bairro Boqueirão foi de 70\%, talvez porque esse bairro possua o maior número de idosos acima de 65 anos de idade; o bairro Aparecida apresentou apenas $10 \%$ e no Embaré o percentual foi de $20 \%$. O evento confusão nas mulheres apresentou homogeneidade, se comparado com o dos homens, e novamente aqui o bairro do Boqueirão apresenta-se em destaque com alto grau de confusão no grupo feminino na tomada das medicações, com o percentual de $54 \%$; já no bairro Aparecida o evento de esquecimento nas mulheres apresentou-se em 13\%; e no bairro Embaré o valor foi de $33 \%$.

Justifica o título do artigo o fato de que outro dado extraído da pesquisa diz respeito ao fato de que principalmente os homens procuram a farmácia antes de procurar um serviço médico diante de algum mal-estar imediato. Da mesma forma, e consonante com isso, são eles que solicitam mais do que as mulheres a indicação de medicamentos não prescritos.

Todas as três farmácias que participaram da pesquisa tinham o farmacêutico no estabelecimento durante todo o horário de funcionamento. As entrevistas com os profissionais abordaram a rotina de trabalho e as informações da empresa. Observouse nesses profissionais a vivência de conflitos em relação à ética profissional contraposta ao objetivo do estabelecimento, ditado pela lógica do mercado. Em consequência, a orientação farmacêutica vê-se fragilizada diante da ausência de mecanismos que fortaleçam a postura do profissional farmacêutico, fundamental para a relação paciente-medicamento, que implica o acompanhamento e a informação correta do paciente, incluindo a avaliação dos fatores de risco, prevenção e promoção da saúde e vigilância das doenças.

As entrevistas realizadas com os farmacêuticos mostraram que existe um grande consumo de medicamentos não prescritos por parte dos idosos, muitas vezes motivados pela crença de conhecer o seu próprio problema de saúde ou pela excessiva e abusiva publicidade desses medicamentos pelos meios de comunicação, ou mesmo pela presença da propaganda de medicamentos no interior das próprias farmácias, a começar pela sua distribuição estratégica nas gôndolas, associada à pressão e persuasão por parte dos laboratórios e desses estabelecimentos para que seus funcionários motivem o cliente a comprar produtos desnecessários.

Quanto à relação entre nível socioeconômico e consumo de medicamentos não prescritos, os dados obtidos reafirmam pesquisas nacionais e internacionais sobre o tema, uma vez que o maior poder aquisitivo de determinados segmentos sociais 
contribui para o acesso a esses medicamentos, essencialmente diante da facilidade da oferta e da pouca ou nenhuma exigência de receituário para sua obtenção. Pesquisas realizadas em países desenvolvidos apontam que a renda dos indivíduos parece não influenciar o autoconsumo por parte dos idosos; no entanto, em estudo realizado na cidade do Rio de Janeiro (Miralles e Kimberlin, 1998), constatou-se um consumo mais reduzido de medicamentos não prescritos, nos bairros mais pobres, enquanto os bairros mais privilegiados apresentaram um consumo alto desses medicamentos. E o mais importante é que essa população, tal como a que fez parte da presente pesquisa,tem facilidade de acesso aos serviços de saúde, apresenta maior frequência regular às consultas, possui mais acesso à informação e renda acima de dois salários mínimos, sendo fatores que poderiam em certa medida inibir essa prática.

Importante também destacar que, no geral, até como resultado dos programas de saúde da mulher, em destaque nas últimas décadas, as mulheres idosas apresentaram maior preocupação com a saúde e mais cuidado ao tomar medicamentos não prescritos, até porque os dados obtidos confirmam que elas constituem o grupo de maior consumo de medicamentos prescritos, relacionados tanto ao autocuidado como à detecção precoce no tratamento de doenças crônicas. Já no que diz respeito aos homens idosos, eles consomem mais medicamentos não prescritos e frequentam as farmácias com maior regularidade do que as mulheres idosas, o que confirma os resultados do estudo de base populacional junto a idosos realizado por Loyola e colegas (2006),na área metropolitana da cidade de Belo Horizonte.

O estudo conseguiu demonstrar que a prática de autoconsumo de medicamentos não prescritos é frequente entre a população idosa, mesmo quando tendo acesso à assistência médica, e mesmo com a possibilidade, que com frequência pode se concretizar, de provocar sintomas indesejáveis após o seu uso, com o potencial de poder provocar danos até mais graves.

O acompanhamento do profissional farmacêutico junto às farmácias é fundamental para que o objetivo do tratamento seja bem-sucedido e venha reduzir o consumo desnecessário, contribuindo para a promoção de seu uso racional.

\section{Conclusão}

O processo de envelhecimento da população brasileira apresenta desafios para as mais variadas áreas do conhecimento. Em relação à saúde, dentre os inúmeros aspectos que devem ser explorados, incluise a farmacoterapia. Faz-se necessária atenção e cuidado para com esse segmento da população, pois a associação medicamentosa, quando provinda do consumo de medicamentos prescritos associado ao de medicamentos não prescritos, apresenta riscos de tal monta que coloca a questão da orientação medicamentosa como um dos principais focos da saúde do idoso. E muito embora o consumo de medicamentos sem indicação médica seja um fenômeno mundial, envolvendo vários fatores e dimensões em sua prática, o que difere a experiência de um país para a do outro são suas políticas de saúde que intervêm e articulam-se com outras políticas públicas no sentido de inibi-la.

A pesquisa permitiu apresentar um perfil de consumo e de prática de medicamentos entre a população idosa de uma região de Santos, apontando fatores externos à relação médico/paciente ou usuário/profissional farmacêutico que conformam esse perfil. Nesse estudo, a população investigada é uma população que tem acesso a medicamentos prescritos e não prescritos de maneira facilitada através da facilidade de acesso aos serviços de saúde e à possibilidade de consumo, por tratar-se de uma população com renda de no mínimo dois salários mínimos. Nessa situação, fatores como propaganda, valores, achar que conhece melhor seu problema de saúde que o profissional médico, além da pressão das indústrias e do comércio do ramo junto a seus próprios funcionários, torna o medicamento uma mercadoria a ser consumida de per si, e não um bem necessário para se conquistar ou promover a saúde. Alerte-se, no entanto, que a presente pesquisa não permitiu relacionar o fato de ir à farmácia comprar medicação sem orientação médica com uma situação pontual, associada, por exemplo, a uma ocorrência de "vir a passar mal"; no entanto, as evidências apontam que essa é uma prática comum, e que portanto merece atenção para que seja minimizada.

Outra evidência a ser destacada é o fato de o idoso, ao comprar o medicamento sem prescrição, 
não solicitar informação ao farmacêutico. Pode-se deduzir então que ele ainda não vê o profissional farmacêutico como parte e extensão dos serviços prestados pela farmácia, já que o cliente tende a associá-la ao "remédio" e não ao serviço de atenção farmacêutica. Constatação nada surpreendente, uma vez que o medicamento é tratado nas farmácias como mera mercadoria de consumo, promovendo sua banalização junto ao consumidor. Como visto, são inúmeros os recursos mercadológicos que contribuem para tanto, e que abrangem desde a propaganda direta ou nos meios de comunicação até a não exigência da apresentação do receituário, a exposição facilitada dos medicamentos nas gôndolas das farmácias e o baixo poder regulador dos órgãos públicos responsáveis.

Consonante com isso chama a atenção o evento muito presente junto a esses consumidores do esquecimento e/ou da confusão na tomada da medicação prescrita e diária, que apresentou valores percentuais altos, sobretudo em se tratando de uma população assistida por médicos, evidenciando que esse fato não impede a procura da farmácia e a compra de outros medicamentos. De forma articulada, a tensão e o conflito em que se encontram os profissionais farmacêuticos que trabalham nas farmácias, sobretudo com relação à tensão inconciliável entre as premissas da ética profissional e os objetivos de mercado do estabelecimento em que trabalha, criam para esses profissionais dificuldades, desconforto, ausência de autonomia, levando o farmacêutico basicamente a uma postura de obediência, subordinada à necessidade da venda dessa mercadoria "remédio", revelando-se frágil diante das forças do comportamento de mercado, a que estão submetidos os próprios idosos na sua qualidade de consumidores.

Fato esse tão mais significativo na medida em que as farmácias de fato desempenham um papel fundamental, embora ainda informal, no processo de atenção à saúde, sendo a presença do profissional farmacêutico nesses estabelecimentos fundamental para que a farmácia se transforme num estabelecimento de saúde, criando laços baseados na relação paciente-farmacêutico visando a busca da otimização de resultados na farmacoterapia em benefício da saúde do paciente. Mas se o acompanhamento farmacêutico junto ao paciente, usuário de medicamentos que os adquire nas farmácias privadas, é primordial para a redução do consumo de medicamentos não prescritos, e se a informação prestada por ele vem afirmar a importância do processo de esclarecimento acerca dos medicamentos e de outros elementos envolvidos na farmacoterapia,esse novo cenário apresenta dois desafios: como inserir esse profissional, com competências sociais e biomédicas específicas, alocado nas farmácias no processo dos cuidados à saúde, e como resolver a talvez insolúvel contradição entre as lógicas pública e privada que envolvem a farmacoterapia no processo de garantias do direito universal à saúde. Nesse caso específico, esse direito é remetido ao segmento idoso da população.

\section{Referências}

BRASIL. Ministério da Saúde. Secretaria de Políticas de Saúde. Departamento de Atenção Básica. Política Nacional de Medicamentos 2001. Brasília, DF, 2001.

BRASIL. Ministério da Saúde Gabinete do Ministro. Portaria $\mathrm{n}^{\mathrm{0}}$. 2.587, de o6 de dezembro de 2004. Institui o incentivo financeiro do Programa Farmácia Popular do Brasil, e dá outras providências. Brasília, 2004. Disponível em: <http://dtr.saude.gov.br/sas/PORTARIAS/ Port2004/Gm/GI>. Acesso em: 15 jun. 2012.

IBGE - INSTITUTO BRASILEIRO DE GEOGRAFIA E ESTATÍSTICA. Censo demográfico. Rio de Janeiro, 2000.

LIMA-COSTA, M. F.; BARRETO, S. M.; GIATTI, L. Condições de saúde, capacidade funcional, uso de serviços de saúde e gastos com medicamentos da população idosa brasileira: um estudo descritivo baseado na Pesquisa Nacional por Amostra de Domicílios. Cadernos de Saúde Pública, Rio de Janeiro, v. 19, n. 3, p. 735-743, 2003.

LOYOLA FILHO, A. I. de; UCHOA, E.; LIMA-COSTA, M. F. Estudo epidemiológico de base populacional sobre uso de medicamentos entre idosos na região metropolitana de Belo Horizonte, Minas Gerais, Brasil. Cadernos de Saúde Pública, Rio de Janeiro, n. 22, n. 12, p. 2657-2667, 2006. 
MIRALLES, M. A.; KIMBERLIN, C. L. Perceived access to care and medication use among ambulatory elderly in Rio de Janeiro, Brazil. Social Science \& Medicine, v. 46, p. 345-355, 1998.

SALDANHA, A. L.; CALDAS, P. C. (Org.). Saúde do idoso: a arte de cuidar. 2. ed. Rio de Janeiro: Interciência, 2004.

SANTOS, J. S. CFF: aspectos desejáveis dos estabelecimentos. Pharmacia Brasileira, Brasília, DF, ano 2, n. 10, p. 24, 1998a.

SANTOS, J. S. Documento contendo um conjunto de alternativas para solucionar problemas agudos do setor farmacêutico. Pharmacia Brasileira, Brasília, DF, ano 2, n. 10, p. 9-11, 1998b.

SEADE - FUNDAÇÃO SISTEMA ESTADUAL DE ANÁLISE DE DADOS. Secretária de Economia e Planejamento. Governo do Estado de São Paulo. S. Paulo, 200o. Estatísticas (1): Seade Índice Paulista de Responsabilidade Social (IPRS). Disponível em: <http://www.novomilenio.info.br/ santos/stestat>. Acesso em: 15 jun. 2012.

ZUBIOLI, A. Pronunciamento proferido no debate técnico sobre assistência farmacêutica, 26 jun. 1996. Pharmacia Brasileira, Brasília, p. 1-2, 1996. Edição especial. 